\title{
Perfil de competências de preceptores para a Atenção Primária em Saúde
}

\author{
Lydiane dos Santos Dantas*; Rebeca Valeska Soares Pereira*; Ítalo de Macedo Bernardino**; Raiany \\ Cristina Pastorelli Paim Figueiredo***; Renata Cardoso Rocha Madruga****; Rilva Suely de Castro \\ Cardoso Lucas****
}

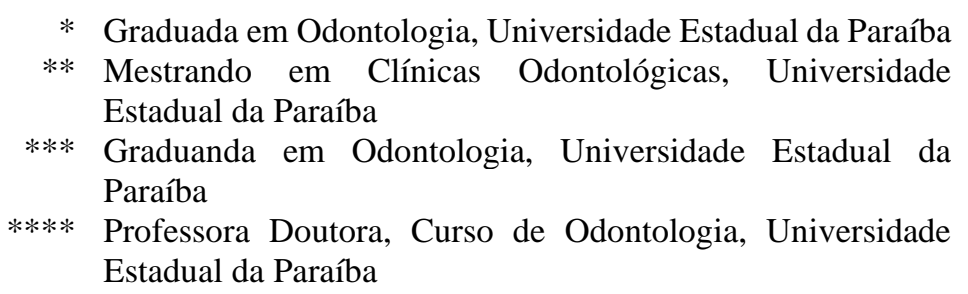

Recebido em 22/05/2018. Aprovado em 07/07/2019.

\begin{abstract}
RESUMO
A integração ensino-serviço propõe uma nova forma de pensar a formação. O papel do preceptor é fundamental, mediando o aprendizado do estudante a partir de vivências nos serviços. O presente estudo transversal objetivou avaliar o perfil de competências dos cirurgiões dentistas (CD) atuantes na Atenção Primária em Saúde no município de Campina Grande quanto ao exercício da preceptoria, entendendo a importância desses resultados para as instituições repensarem a formação e proporcionar à gestão o conhecimento do perfil de profissionais que integram sua rede de assistência. Os dados foram coletados por meio de questionário e então submetidos a análise estatística descritiva, bem como empregou-se a análise de diferença de proporções (teste qui-quadrado de Pearson ou teste exato de Fisher, quando apropriado) para investigar associações entre as variáveis qualitativas estudadas. A maior parte assinalou que a integração ensino-serviço na formação do CD é extremamente importante $(\mathrm{n}=19 ; 44,2 \%)$. Uma parcela expressiva ainda não participou de formação para preceptores $(n=31 ; 72,1 \%)$, constatou-se associação estatisticamente significativa entre já ter participado de alguma formação e relato de sentir-se preparado para a função $(p=0,005)$. Foram relatadas dificuldades no processo de desenvolvimento da preceptoria $(\mathrm{n}=32 ; 74,4 \%)$, destacandose falta de programa de capacitação $(n=29 ; 67,4 \%)$. Os resultados apontam para a necessidade de processos formativos para os preceptores, que contribuam para uma reflexão consistente sobre o modelo de atenção à Saúde e formação de novos profissionais.
\end{abstract}

Descritores: Preceptoria. Saúde Pública. Recursos Humanos em Odontologia. 


\section{INTRODUÇÃO}

A saúde no Brasil sofreu fortes mudanças nas décadas de 1980 e 1990, com a instituição de um sistema universal, o Sistema Único de Saúde (SUS), criado pela Constituição de 1988 e regulamentado por Leis Orgânicas de Saúde (8.080/90 e 8142/90). Considerado uma das maiores conquistas sociais do Brasil, representa a materialização de uma nova concepção, que precisa ser apropriada pelas Instituições de Educação Superior (IES) ${ }^{1}$.

O modelo de ordenação do cuidado escolhido pelo Ministério da Saúde foi o da Atenção Primária em Saúde (APS). No Brasil, sua operacionalização ocorreu em 1994, com a escolha do Programa Saúde da Família (PSF), posteriormente, em 2006, chamado Estratégia Saúde da Família $(\mathrm{ESF})^{2}$, da qual o cirurgião dentista (CD) só veio fazer parte em 2001, com a publicação da Portaria $1444^{3}$. No entanto, a apropriação do contexto determinante do processo saúde-doença e uma assistência generalista e humanista prescindem vivências nos serviços de saúde durante o período de formação desses profissionais ${ }^{4}$.

Diante dos desafios impostos pelo novo modelo de formação, suscitaram-se discussões acerca de mudanças na graduação ${ }^{5}$. Assim, foi proposta, em 1996, a Lei de Diretrizes e Bases da educação $^{6}$, que rege a educação brasileira, propondo, dentre outras medidas, a substituição dos currículos mínimos pelas Diretrizes Curriculares Nacionais (DCN) para os cursos de graduação $^{7,8}$.

Dentro das demandas de inserção precoce do graduando em seu contexto profissional e da diversificação dos cenários de aprendizagem, a "Integração Ensino-Serviço" encontra um lócus privilegiado para a reflexão sobre a realidade da produção de cuidados ${ }^{9}$. Em tal cenário, a preceptoria em saúde mostra-se como um fator de grande importância, possibilitando a inserção precoce do estudante no ambiente de assistência à saúde, permitindo a criação de possibilidades para que ele se desenvolva e termine o ensino superior com um perfil de formação diferenciado ${ }^{10}$.

Desta forma, a presente pesquisa propôs avaliar o perfil de competências dos Cirurgiões Dentistas atuantes na APS no município de Campina Grande/PB, quanto ao exercício da preceptoria, entendendo a importância desses resultados para as IES repensarem a formação e proporcionar à gestão o conhecimento do perfil de profissionais que integram sua rede de assistência.

\section{MATERIAL E MÉTODOS}

Tratou-se de um estudo quantitativo e analítico, com um desenho do tipo transversal. A pesquisa foi desenvolvida na rede de Atenção Primária em Saúde do município de Campina Grande/PB.

Conforme a literatura ${ }^{11}$, por razões de natureza estatística, toda vez que o tamanho da população de referência for inferior ou igual a 250 indivíduos, recomenda-se a realização de um censo, ou seja, examina-se toda a população. De acordo com dados fornecidos pela Secretaria Municipal de Saúde existiam, na ocasião da pesquisa, 57 equipes de Saúde Bucal credenciadas no município. No entanto, 13 destas equipes se encontravam sem $\mathrm{CD}$, totalizando 44 equipes de Saúde Bucal em funcionamento na ESF do município.

Foram incluídos no estudo todos os CD atuantes no nível de APS, independente de idade e sexo, que concordaram em participar da pesquisa, assinando o Termo de Consentimento Livre e Esclarecido (Resolução 466/2012); excluídos aqueles que não se encontravam em exercício pleno da função durante o período da pesquisa e considerado perdas os profissionais que não foram encontrados nos respectivos locais de trabalho, após três retornos consecutivos, em dias e horários alternados, ou recusaram-se a participar da 
pesquisa.

O estudo seguiu a normativa da Resolução No 466/2012 do Conselho Nacional de Saúde que regulamenta a pesquisa em seres humanos, obtendo parecer favorável do Comitê de Ética em Pesquisa da Universidade Estadual da Paraíba (CAAE: 63009916.7.0000.5187).

$\mathrm{O}$ instrumento de pesquisa com vistas a avaliar o perfil de competências dos $\mathrm{CD}$ quanto ao exercício da preceptoria foi construído a partir das DCN para os cursos da área da saúde aprovadas em $2002^{7}$. Partindo dos conhecimentos e competências estabelecidas e necessárias para que a formação superior seja reordenada conforme as necessidades do SUS, foram elaboradas 20 questões objetivas, que foram agrupadas, por conveniência, em quatro dimensões: caracterização dos profissionais; integração ensino-serviço; atividade de preceptoria e relação com o estagiário.

O controle de qualidade dos dados foi realizado por meio de análise de reprodutibilidade (re-teste), que tem por finalidade medir o grau de correlação e concordância das respostas dadas em dois momentos diferentes e validade dos dados coletados. Para realizar esta avaliação 10 sujeitos participantes, sorteados aleatoriamente, foram entrevistados uma segunda vez, no prazo entre dois e sete dias após aplicação do primeiro questionário, utilizando as correlações de Spearman e Pearson para variáveis ordinais e a aplicação do teste Kappa, para variáveis dicotômicas.

Os dados obtidos foram submetidos à análise por meio da estatística descritiva, sendo calculadas as frequências absolutas e percentuais para as variáveis qualitativas, bem como, as medidas de tendência central (média, mediana) e de variabilidade (desvio-padrão, valor mínimo, valor máximo) para as variáveis quantitativas.

Empregou-se a análise de diferença de proporções (teste qui-quadrado de Pearson ou teste exato de Fisher, quando apropriado) para investigar associações entre as variáveis qualitativas estudadas $^{12}$. O nível de significância foi fixado em $5 \%(\mathrm{p}<0,05)$. Todas as análises foram feitas usando o software IBM SPSS Statistics versão 20.0 e considerando um intervalo de confiança de $95 \%$.

\section{RESULTADOS E DISCUSSÃO}

Considerando que o estudo abrangeu todos os CD atuantes no nível de APS do município de Campina Grande, a relevância dos resultados traz um alerta para as IES e para a gestão dos serviços públicos quanto ao o perfil dos profissionais que assumem a função de preceptor na rede de serviços, permitindo que seja feita a reflexão sobre a necessidade de processos de formação para a preceptoria, considerando a importância do preceptor na formação dos estudantes, papel que só será exercido com efetividade se todas as competências exigidas forem seguramente consolidadas.

A tabela 1 mostra a distribuição dos participantes de acordo com as características sociodemográficas, perfil de formação e atuação profissional. A maioria era do sexo feminino $(\mathrm{n}=$ $39 ; 90,7 \%$ ), tinha até 45 anos de idade ( $\mathrm{n}=24$; $55,8 \%$ ), e relatou ter se formado há 20 anos ou menos $(n=23 ; 53,5 \%)$.

Quanto ao sexo, os resultados foram compatíveis com os da literatura ${ }^{13,14}$, que traz a feminização das profissões como uma tendência das áreas da saúde, devido à expansão e à elevação dos níveis de escolaridade e instrução, bem como as mudanças econômicas ocorridas no Brasil, que propiciaram a formação de um mercado de trabalho aberto para a mulher ${ }^{15}$.

A tabela 2 mostra a distribuição dos participantes de acordo com as percepções sobre a integração ensino-serviço. A maior parte assinalou que a integração ensino-serviço na formação do CD é extremamente importante $(n=19 ; 44,2 \%)$. No entanto, uma parcela expressiva dos profissionais ainda não participou de formação para preceptores $(n=31 ; 72,1 \%)$. Essa importância 
se potencializa no contexto de mudanças na formação de CD no Brasil, exigindo uma educação integral para o SUS, oportunizando o aprendizado e trabalho em todos os espaços que compõem a
Rede de Atenção à Saúde ${ }^{16}$. Diante dessa realidade, uma das propostas das DCN é a inclusão de estágios no serviço público desde os períodos iniciais da graduação ${ }^{17,18}$.

Tabela 1. Distribuição dos participantes de acordo com as características sociodemográficas, tempo de formação e atuação profissional

\begin{tabular}{lcc}
\hline \multicolumn{1}{c}{ Variáveis } & $\mathbf{n}$ & \% \\
\hline Idade $(\text { em anos })^{*}$ & & \\
$\leq 45$ anos & 24 & 55,8 \\
$>45$ anos & 19 & 44,2 \\
Sexo & & \\
Masculino & 4 & 9,3 \\
Feminino & 39 & 90,7 \\
Tempo de formação (em anos)* & & \\
$\leq 20$ anos & 23 & 53,5 \\
> 20 anos & 20 & 46,5 \\
\hline *ariáveis dicotomizadas pela mediana. & &
\end{tabular}

Tabela 2. Distribuição dos participantes de acordo com as percepções sobre a integração ensinoserviço

\begin{tabular}{lcc}
\multicolumn{1}{c}{ Variáveis } & n & $\%$ \\
\hline Como você avalia a integração ensino-serviço (estagiários na rede do & & \\
SUS) na formação do cirurgião-dentista? & 0 & 0,0 \\
Sem importância & 0 & 0,0 \\
Pouco importante & 8 & 18,6 \\
Importante & 16 & 37,2 \\
Muito importante & 19 & 44,2 \\
Extremamente importante & & \\
Você acredita que a atividade de preceptoria realmente é importante & \\
na formação do aluno? & 43 & 100,0 \\
Sim & 0 & 0,0 \\
Não & 12 & 27,9 \\
Você já participou de alguma formação para preceptores? & 31 & 72,1 \\
Sim & & \\
Não & 24 & 55,8 \\
Você sente-se preparado para a função de preceptoria? & 19 & 44,2 \\
Sim & & \\
Não & & \\
& & \\
\end{tabular}

A literatura internacional destaca os serviços de saúde enquanto espaços de formação nos currículos dos cursos de Odontologia ${ }^{19-22}$, os quais aproximam o estudante da profissão inserida em sua realidade, colocando-o em contato com as práticas e políticas em saúde pública, de forma a identificar sua posição no SUS ${ }^{17,23-27}$.

Todos os profissionais afirmam ter $\mathrm{o}$ 
conhecimento do significado da preceptoria. De acordo com a literatura, os preceptores são profissionais vinculados aos serviços do SUS, que recebem estudantes de graduação ou pósgraduação da área da saúde, no contexto de um programa de educação, a fim de acompanhá-los e orientá-los, possibilitando a apropriação de conhecimentos, habilidades e atitudes importantes para sua atuação profissional, estreitando a distância entre a teoria e a prática ${ }^{28,29}$. É na perspectiva da preceptoria que o trabalho é considerado como princípio educativo, valorizando os saberes provenientes da experiência de trabalho na formação de outros profissionais ${ }^{17,30}$.

Os dados evidenciaram que todos os $\mathrm{CD}$ reconhecem que o exercício de preceptoria é realmente importante para o estudante, porém foi verificado que uma parcela expressiva dos respondentes ainda não participou de formação destinada a esse fim, sendo encontrada associação estatisticamente significativa entre já ter participado e sentir-se mais preparado para a função $(p=0,05) \quad($ tabela 3$)$, apontando a necessidade de cursos de formação para os $\mathrm{CD}$ atuarem na preceptoria, entendidos como imprescindíveis para a aquisição e consolidação de competências necessárias para esse fim $^{31}$.

Considerando a associação evidenciada pela pesquisa é válido ressaltar que um dos pressupostos apontados pela Política Nacional de Saúde Bucal é a definição de uma política de educação permanente para os trabalhadores, a fim de implementar projetos de mudança na formação técnica da graduação e pós-graduação ${ }^{32,33}$, considerando a responsabilidade e compromisso desses com as futuras gerações de profissionais, como preconizado pelas DCN para os cursos de Odontologia ${ }^{7}$.

No entanto, um dos desafios a serem superados é justamente o que diz respeito ao papel, atribuições, perfil e institucionalizações do profissional preceptor ${ }^{34}$. A inserção de estudantes na rede de serviços evidencia alguns debates de ordem pedagógica, principalmente no que diz respeito a problemas vivenciados pelos trabalhadores de saúde em seu cotidiano, especificamente quanto às políticas de recursos humanos e à inexistência de apoio institucional e oportunidades para acesso à cursos de formação e educação continuada ${ }^{27,35,36}$.

A tabela 4 mostra a distribuição dos participantes de acordo com as percepções sobre a atividade de preceptoria e a relação com o estagiário. A maioria relatou que atua ou já atuou como preceptor $(\mathrm{n}=26 ; 60,5 \%)$, bem como, existem dificuldades no processo de desenvolvimento da preceptoria $(n=32 ; 74,4 \%)$. As três dificuldades mais apontadas foram: falta de insumos $(\mathrm{n}=34 ; 79,1 \%)$, falta de programa de capacitação para atuar na preceptoria em saúde (n $=29 ; 67,4 \%)$ e falta de incentivo financeiro $(n=23$; $53,5 \%)$.

Tabela 3. Associação entre sentir-se preparado para a função de preceptoria e a participação prévia em alguma formação para preceptores.

\begin{tabular}{|c|c|c|c|c|}
\hline \multirow{2}{*}{ Variável } & \multicolumn{3}{|c|}{$\begin{array}{c}\text { Sente-se preparado para a função de } \\
\text { preceptoria? }\end{array}$} & \multirow[b]{2}{*}{ p-valor } \\
\hline & $\underset{\mathbf{n}(\%)}{\operatorname{Sim}}$ & $\begin{array}{l}\text { Não } \\
\text { n }(\%)\end{array}$ & $\begin{array}{l}\text { Total } \\
\text { n }(\%)\end{array}$ & \\
\hline \multicolumn{4}{|c|}{ Você já participou de alguma formação para preceptores? } & $0,005 *$ \\
\hline Sim & $11(45,8)$ & $1(5,3)$ & $12(27,9)$ & \\
\hline Não & $13(54,2)$ & $18(94,7)$ & $31(72,1)$ & \\
\hline
\end{tabular}

Teste exato de Fisher. $* \mathrm{p}<0,05$. 
Tabela 4. Distribuição dos participantes de acordo com as percepções sobre a atividade de preceptoria e a relação com o estagiário

\begin{tabular}{lcc}
\multicolumn{1}{c}{ Variáveis } & $\mathbf{n}$ & $\mathbf{\%}$ \\
\hline Você atua ou já atuou como preceptor? & & \\
Sim & 26 & 60,5 \\
Não & 17 & 39,5 \\
Existem dificuldades no processo de desenvolvimento da preceptoria? & & \\
Sim & 32 & 74,4 \\
Não & 11 & 25,6 \\
Das alternativas a seguir, quais você considera como dificuldade para o & & \\
exercício da preceptoria?* & \multicolumn{2}{c}{} \\
Falta programa de capacitação para atuar na preceptoria em saúde & 29 & 67,4 \\
Falta comprometimento dos estudantes & 4 & 9,3 \\
Desperdício de tempo clínico com orientação aos alunos & 3 & 7,0 \\
Demanda alta de alunos & 4 & 9,3 \\
Aumento do volume de trabalho & 8 & 18,6 \\
Falta de incentivo financeiro & 23 & 53,5 \\
Falta de apoio da instituição de onde os estudantes advêm & 11 & 25,6 \\
Falta de insumos (instrumentais, EPI's, materiais educativos, etc) & 34 & 79,1 \\
\hline
\end{tabular}

* O participante podia assinalar mais de uma alternativa

Os dados encontrados em nosso estudo foram condizentes com as investigações de outros autores ${ }^{37-40}$, os quais mostraram ser barreiras para a preceptoria o desenho das unidades não preparadas para o estagiário, as condições de precarização vividas no ambiente de trabalho, desconforto do preceptor com o papel de professor, curta experiência na função de preceptoria, déficit na educação permanente dos profissionais, efeito prejudicial sobre a produtividade $\mathrm{e}$ falta de incentivo financeiro ${ }^{37}$, o que fortalece o argumento daqueles que não se interessam pela inserção dos estudantes no serviço, trazendo reflexos, inclusive, de experiências negativas anteriores.

Todas as competências abordadas no instrumento da pesquisa foram traçadas conforme as DCN para o curso de Odontologia ${ }^{7}$. Quanto às percepções do profissional preceptor sobre elas, o respeito aos princípios da bioética e ética profissional $(\mathrm{n}=20 ; 76,9 \%)$ e a atualização constante, entendendo a importância de novos conhecimentos e dos estágios para as futuras gerações de profissionais $(n=18 ; 69,2 \%)$ foram avaliadas como "extremamente importantes" (tabela 5).

Carvalho, Ventura, Barroso ${ }^{41}$ ao analisar um conjunto de competências necessárias ao preceptor na área médica, relataram que são importantes responsabilidade e ética profissional, segurança técnica e capacidade crítica, de comunicação, de gerir os recursos e, por fim, competências pedagógicas. Pela natureza das relações estabelecidas no processo de aprendizagem, os preceptores também são vistos como modelos para o crescimento profissional dos estudantes, tendo, portanto, total responsabilidade na formação ética dos mesmos ${ }^{39}$. 
Tabela 5. Distribuição dos participantes de acordo com as percepções sobre as competências que caracterizam um bom perfil de preceptor

\begin{tabular}{|c|c|c|}
\hline+3 & $\mathbf{n}$ & $\%$ \\
\hline \multicolumn{3}{|c|}{ Aptidão de desenvolvimento de atribuições em seu nível de saúde. } \\
\hline Sem importância & 0 & 0,0 \\
\hline Pouco importante & 0 & 0,0 \\
\hline Importante & 5 & 19,2 \\
\hline Muito importante & 14 & 53,8 \\
\hline Extremamente importante & 7 & 26,9 \\
\hline \multicolumn{3}{|l|}{ Prática de forma integrada, resolutiva e contínua } \\
\hline Sem importância & 0 & 0,0 \\
\hline Pouco importante & 0 & 0,0 \\
\hline Importante & 4 & 15,4 \\
\hline Muito importante & 11 & 42,3 \\
\hline Extremamente importante & 11 & 42,3 \\
\hline \multicolumn{3}{|l|}{ Habilidade para tomada de decisões } \\
\hline Sem importância & 0 & 0,0 \\
\hline Pouco importante & 0 & 0,0 \\
\hline Importante & 2 & 7,7 \\
\hline Muito importante & 16 & 61,5 \\
\hline Extremamente importante & 8 & 30,8 \\
\hline \multicolumn{3}{|c|}{ Respeito aos princípios da bioética e ética profissional } \\
\hline Sem importância & 0 & 0,0 \\
\hline Pouco importante & 0 & 0,0 \\
\hline Importante & 1 & 3,8 \\
\hline Muito importante & 5 & 19,2 \\
\hline Extremamente importante & 20 & 76,9 \\
\hline \multicolumn{3}{|l|}{ Acessibilidade } \\
\hline Sem importância & 0 & 0,0 \\
\hline Pouco importante & 0 & 0,0 \\
\hline Importante & 1 & 3,8 \\
\hline Muito importante & 13 & 50,0 \\
\hline Extremamente importante & 12 & 46,2 \\
\hline \multicolumn{3}{|l|}{ Capacidade de liderar, administrar e gerenciar } \\
\hline Sem importância & 0 & 0,0 \\
\hline Pouco importante & 0 & 0,0 \\
\hline Importante & 2 & 7,7 \\
\hline Muito importante & 16 & 61,5 \\
\hline Extremamente importante & 8 & 30,8 \\
\hline \multicolumn{3}{|l|}{ Atualização constante } \\
\hline Sem importância & 0 & 0,0 \\
\hline Pouco importante & 0 & 0,0 \\
\hline Importante & 0 & 0,0 \\
\hline Muito importante & 8 & 30,8 \\
\hline Extremamente importante & 18 & 69,2 \\
\hline
\end{tabular}




\section{CONCLUSÃO}

Os CD atuantes na APS de Campina Grande são, predominantemente, do sexo feminino, com 20 anos ou menos de formados, sendo que a maioria já atuou como preceptor e considera a integração ensino-serviço importante na formação do estudante. No entanto, a falta de programas de capacitação para atuar na preceptoria em saúde está entre uma das principais dificuldades relatadas, apontando a necessidade de processos de formação. A temática pesquisada não se esgota com os resultados apresentados por esta pesquisa, são imprescindíveis outros estudos que busquem contribuir para a compreensão do papel e perfil do preceptor, reconhecendo-o como imprescindível no âmbito do ensino e do serviço.

\section{ABSTRACT \\ Profile of competences of preceptors for Primary Health Care}

The teaching-service integration proposes a new way of thinking about training. The role of the preceptor is fundamental, mediating the student's learning from experiences in health services. This cross - sectional study aimed to evaluate the profile of competences of dental surgeons (DS) working in the Primary Health Care in the city of Campina Grande regarding the exercise of preceptorship, understanding the importance of these results for institutions to rethink training and provide management with knowledge of the profile of professionals that integrate their assistance network. Data were collected by means of a questionnaire and submitted to descriptive statistical analysis, and analysis of proportional difference (Pearson's chi-square test or Fisher's exact test, when appropriate) was used to investigate associations between qualitative variables. Most indicated that the teachingservice integration in DS formation is extremely important $(\mathrm{n}=19,44.2 \%)$. A significant proportion have not yet participated in training for preceptors $(\mathrm{n}=31 ; 72.1 \%)$; statistically significant association was found between having participated in some training and reporting feeling prepared for the function $(\mathrm{p}=$
0.005). Difficulties were reported in the development of preceptorship $(\mathrm{n}=32 ; 74.4 \%)$, highlighting lack of training programs $(\mathrm{n}=29$; $67.4 \%$ ). Results point to the need for training processes for preceptors, which contribute to a consistent reflection on the health care model and the training of new professionals.

Descriptors: Preceptorship. Public health. Human Resources in Dentistry.

\section{REFERÊNCIAS}

1. Gonzaléz AD, Almeida MJ. Movimentos de mudança na formação em saúde: da medicina comunitária às diretrizes curriculares. Physis 2010; (20)2: 551-70.

2. Maeyama MA, Machado C, Cutolo LRA, Ceretta LB, Mattos DB, Bevilacqua LL. Integração ensino-serviço na atenção básica: uma proposta de instrumento de avaliação. Inova Saúde 2016; (5)1:1-29.

3. Giovanella L, Mendonça MHM, Almeida PF, Escorel S, Senna MCM, Fausto MCR et al. Saúde da família: limites e possibilidades para uma abordagem integral de atenção primária à saúde no Brasil. Ciênc Saúde Colet. 2009;(14)3:783-94.

4. Maciel JAC, Vasconcelos MIO, CastroSilva II, Teixeira AKM, Farias MR, Queiroz MVO. A integração ensino-serviço em odontologia: Uma experiência na atenção primária à saúde no município de Sobral, Ceará. Rev APS 2016; (19)4:650-5.

5. Nogueira MI. As mudanças na educação médica brasileira em perspectiva: reflexões sobre a emergência de um novo estilo de pensamento. Rev Bras Educ Méd 2009; (33) 2:262-70.

6. Brasil. Senado Federal. LDB: Lei de Diretrizes e Bases da Educação. Brasília: Senado Federal, Coordenação de Edições Técnicas, 2017, 58p. [Acesso em 16 de junho de 2017]. Disponível em: https://www2.senado.leg.br/bdsf/bitstream/ 
handle/id/529732/lei_de_diretrizes_e_bases 1ed.pdf?sequence $=1$.

7. Brasil. Conselho Nacional de Educação. Câmara de Educação Superior. Resolução CNE/CES 3/2002, de 19 de fevereiro de 2002. Diretrizes Curriculares Nacionais do Curso de Graduação em Odontologia. Diário Oficial da União, Brasília, 04 de março de 2002, seção 1, p.10. [Acesso em 16 de junho de 2017]. Disponível em: http://portal.mec.gov.br/cne/arquivos/pdf/C ES032002.pdf.

8. Luz GW, Toassi RFC. Percepções sobre o preceptor cirurgião-dentista da Atenção Primária à Saúde no ensino da Odontologia. Rev ABENO 2016; (16)1:2-12.

9. Albuquerque VS, Gomes AP, Rezende CHA, Sampaio MX, Dias OV, Lugarinho RM. A integração ensino-serviço no contexto dos processos de mudança na formação superior dos profissionais da saúde. Rev Bras Educ Méd 2008; 32(3):35662.

10. Dias ARN, Paranhos ACM, Teixeira RC, Domingues RJS, Kietzer KS, Freitas JJS. Preceptoria em saúde: percepções e conhecimento dos preceptores de uma unidade de ensino e assistência. Educ Online 2015 (1)19 83-99.

11. Antunes JLF, Peres MA. Epidemiologia da Saúde Bucal. $2^{a}$ ed. São Paulo: Editora Santos, 2013. 738p.

12. Larson, R.; Farber, B. Estatística Aplicada. 6. ed. São Paulo: Pearson Prentice Hall, 2016.

13. Vilarinho SMM, Mendes RF, Prado Júnior RR. Perfil dos cirurgiões dentistas integrantes do Programa Saúde da Família em Teresina (PI). Rev Odonto Ciênc 2007; (22) $55: 48-54$.

14. Santos KT, Ferreira L, Batista RJ, Bitencourt CTF, Araújo RP, Carvalho RB. Percepção discente sobre a influência de estágio extramuros na formação acadêmica odontológica. Rev Odontol UNESP 2013; (42)6:420-5.

15. Pinto ESG, Menezes RMP, Villa TCS. Situação de trabalho dos profissionais da Estratégia Saúde da Família em CearáMirim. Rev Esc Enferm USP 2010; (44)3:657-64.

16. Fonseca EP. As diretrizes curriculares nacionais e a formação do cirurgião-dentista brasileiro. JMPHC 2012; (3)2:158-78.

17. Werneck MAF, Senna MIB, Drumond MM, Lucas SD. Not everything is supervised training: contributions for the debate. Ciênc Saúde Colet. 2010; (15)1:221-31.

18. Grande IMP, Prochnow R, Saab R, Pizzatto E. Desafios na formação do cirurgiãodentista para o SUS. Rev ABENO 2016; (16)3:2-6.

19. Ploteau S, Winer N, Barrier J, Rogez JM, Philippe HJ. Évaluation des enseignements et des enseignants par les externs lors d'un stage clinique dans un service de gynécologie-obstétrique. J Gynecol Obstet Biol Reprod 2007; (36)8:807-16.

20. Hood JG. Service learning in dental education: meeting needs and challenges. J Dent Educ. 2009; (73)4:454-63.

21. Margo K, Gazewood J, Jerpbak C, Burge S, Usatine R. Clerkship directors' characteristics, scholarship, and support: a summary of published surveys from seven medical specialties. Teach Learn Medicine 2009;(21)2:94-9.

22. Davidson PL, Nakazono TT, Carreon DC, Guiterrez JJ, Shahedi S, Andersen RM. Reforming dental workforce education and practice in the USA. Eur J Dent Educ. 2011; (15)2:73-9.

23. Gonçalves CM, Santos KT, Carvalho RB. O PET-Saúde como instrumento de 
reorientação do ensino em Odontologia: a experiência da Universidade Federal do Espírito Santo. Rev ABENO 2011; (11)2:27-33.

24. Oliveira Sobrinho TA, Medeiros CPP, Maia MR, Reias TC, Miranda LP, Costa PF. Integração acadêmica e multiprofissional no PET-Saúde: Experiências e desafios. Rev ABENO 2011; (11)1: 39-42.

25. Albuquerque e Silva T, Junqueira SR. Cenários de prática no Sistema Único de Saúde e as Diretrizes Curriculares Nacionais para o curso de Odontologia. IJHE 2016;1(2)107-13.

26. Barbosa FTL, Teixeira SR, Nunes MF, Freire MCM. Implantação das Diretrizes Curriculares Nacionais nos cursos de Odontologia: opinião de formandos de uma universidade pública. Rev ABENO 2016;(16)4:61-71.

27. Faé JM, Silva Junior MF, Carvalho RB, Esposti CDD, Pacheco KTS A integração ensino-serviço em Odontologia no Brasil. Rev ABENO 2016; (16)3:7-18.

28. Barreto VHL, Monteiro ROS, Magalhães GSG, Almeida RCC, Souza LN. Papel do preceptor da atenção primária em saúde na formação da graduação e pós-graduação da Universidade Federal de Pernambuco: um termo de referência. Rev Bras Educ Méd 2011; (35)4:578-83.

29. Toassi RFC, Davoglio RS, Lemos VMA. Integração ensino-serviço-comunidade: o estágio na atenção básica da graduação em Odontologia. Educ Rev. 2012; (28)4:22342.

30. Rocha PF, Warmling CM, Toassi RFC. Preceptoria como modalidade de ensino na Saúde: Atuação e características do preceptor cirurgião-dentista da atenção primária. Saberes Plurais: Educ Saúde 2016; (1)1: 96-112.
31. Rocha HC, Ribeiro VB. Curso de Formação Pedagógica para Preceptores do Internato Médico. Rev Bras Educ Méd. 2012; (36)3:343-50.

32. Brasil. Ministério da Saúde. Portaria 198/GM/MS. Diário Oficial da União ${ }^{\circ}$ 32/2004, Secção I.

33. Brasil. Ministério da Saúde. Portaria GM/MS n. 198/04, de 13 de fevereiro de 2004: institui a Política Nacional de Educação Permanente em Saúde como estratégia do Sistema Único de Saúde para a formação e o desenvolvimento de trabalhadores para o setor e dá outras providências. Brasília: 2004.

34. Warmlimg CM, Rossoni E, Hugo FN, Toassi RFC, Lemos VA, Slavutzki SMB et al. Estágios curriculares no SUS: experiências da Faculdade de Odontologia da UFRGS. Rev ABENO 2011; (11)2:63-70.

35. Trajman A, Assunção N, Venturi M, Tobias D, Toschi W, Brant V. A preceptoria na rede básica da Secretaria Municipal de Saúde do Rio de Janeiro: opinião dos profissionais de Saúde. Rev Bras Educ Méd 2009; (33)1:2432.

36. Montanha M, Peduzzi M. Educação Permanente em Enfermagem: levantamento de necessidades e resultados esperados segundo a concepção dos trabalhadores. Rev Esc Enferm USP. 2010; (44)3:597-604.

37. Peters AS, Schnaidt KN, Zivin K, RifasShiman SL, Katz HP. Important is money as a reward for teaching? Acad Med. 2009; (84) 1:42-6.

38. Barker ER, Pittman O. Becoming a super preceptor: a practical guide to preceptorship in today's clinical climate. J Am Acad Nurse Pract. 2010; (22)3:14449.

39. Botti SHO, Rego S. Docente-clínico: o complexo papel do preceptor na residência médica. Physis 2011;(21)1: 65-85. 
40. Autonomo FROM, Hortale VA, Santos GB, Botti SHO. A preceptoria na formação médica e multiprofissional com ênfase na atenção primária - análise das publicações brasileiras. Rev Bras Educ Méd 2015; (39)2: 316-327.

41. Carvalho F, Ventura T, Barroso R. Perfil de competências do orientador de formação. Rev Port Clin Geral 2004; 20:147-52.

\section{Correspondência para:}

Lydiane dos Santos Dantas

e-mail: 1ydiane.dantaas@gmail.com

Rua Joaquim Pacífico Dantas, 116

58.187-000 Picuí/PB 